\title{
Indoxyl sulfate, a representative uremic toxin, suppresses erythropoietin production in a HIF-dependent manner
}

\author{
Chih-Kang Chiang ${ }^{1,2}$, Tetsuhiro Tanaka ${ }^{1}$, Reiko Inagi ${ }^{1}$, Toshiro Fujita ${ }^{1}$ and Masaomi Nangaku ${ }^{1}$
}

\begin{abstract}
Advanced chronic kidney disease (CKD) patients encounter anemia through insufficient erythropoietin (EPO) production by peritubular fibroblasts. Recent studies showed an increase in EPO production by pharmacological activation of hypoxia-inducible transcription factors (HIFs) in dialysis patients, suggesting that desensitization of the oxygen-sensing mechanism is responsible for the development of renal anemia. Our recent work demonstrated that indoxyl sulfate (IS), a uremic toxin, dysregulates oxygen metabolism in tubular cells. Here we provide evidence of an additional property that IS impairs oxygen sensing in EPO-producing cells. $\mathrm{HepG} 2$ cells were stimulated with cobalt chloride $\left(\mathrm{CoCl}_{2}\right)$ or hypoxia under varying concentrations of IS. EPO mRNA was evaluated by quantitative PCR. Nuclear accumulation of HIF- $\alpha$ was evaluated by western blotting. Transcriptional activity of HIF was checked by hypoxia-responsive element (HRE)-luciferase reporter assay. The impact of IS was further evaluated in vivo by administering rats with indole, a metabolic precursor of $I S$, and subjecting them to $\mathrm{CoCl}_{2}$ stimulation, in which renal EPO mRNA as well as plasma EPO levels were measured by quantitative PCR and enzyme-linked immunosorbent assay, respectively. Although IS induced cellular toxicity at relatively high concentrations $(\geqslant 2.5 \mathrm{mM})$, EPO mRNA expression was significantly suppressed by IS at concentrations below cytotoxic ranges. In HepG2 cells, IS treatment decreased nuclear accumulation of HIF- $\alpha$ proteins and suppressed HRE-luciferase activity following hypoxia. Furthermore, administration of rats with indole suppressed renal EPO mRNA expression and plasma EPO levels, corroborating in vitro findings. Results of the present study provide a possible connection between a uremic toxin and the desensitization of the oxygen-sensing mechanism in EPO-producing cells, which may partly explain inadequate EPO production in hypoxic kidneys of CKD patients.
\end{abstract}

Laboratory Investigation (2011) 91, 1564-1571; doi:10.1038/labinvest.2011.114; published online 22 August 2011

KEYWORDS: chronic kidney disease; erythropoietin; hypoxia-inducible factor; indoxyl sulfate; uremic toxin

High percentage of the chronic kidney disease (CKD) population encounter anemia due to insufficient erythropoietin (EPO) production by the diseased kidney, ${ }^{1,2}$ and are in need of a therapy using erythropoiesis-stimulating agents. Expression of EPO is controlled by hypoxia-inducible transcription factor (HIF), which is a heterodimeric transcription factor composed of a hypoxia-inducible $\alpha$-subunit and a constitutively expressed $\beta$-subunit. The expression of HIF- $\alpha$ (HIF- $1 \alpha$ and HIF- $2 \alpha$ ) is strictly dependent on hypoxia, because molecular oxygen allows the $\mathrm{Fe}^{2+}$-and 2-oxoglutaratedependent prolyl-4 hydroxylase domain (PHD) enzymes to hydroxylate conserved proline residues of HIF- $\alpha$, which triggers recruitment of the von Hippel-Lindau tumor suppressor protein, a component of E3 ubiquitin ligase complex, and facilitates proteasomal degradation of HIF- $\alpha^{3,4}$ Recently, Bernhardt et al ${ }^{5}$ conducted a phase 1 clinical trial of a PHD inhibitor to enhance HIF availability in hemodialysis patients. They found that inhibition of PHD enzymes significantly enhanced EPO production in ESRD patients and suggested that inadequate responses of EPO-producing cells, presumably as a result of desensitization of the oxygensensing mechanism, rather than destruction of the cells per se, may cause the inappropriately low production of EPO in patients with ESRD.

In general, ESRD patients as well as advanced CKD patients are exposed to uremic toxins, even after initiation of

\footnotetext{
${ }^{1}$ Division of Nephrology and Endocrinology, University of Tokyo School of Medicine, Tokyo, Japan and ${ }^{2}$ Department of Internal Medicine, National Taiwan University Hospital, College of Medicine, National Taiwan University, Taipei, Taiwan, ROC

Correspondence: Dr M Nangaku, MD, PhD, Division of Nephrology and Endocrinology, University of Tokyo School of Medicine, 7-3-1 Hongo, Bunkyo-ku,

Tokyo 113-8655, Japan.

E-mail: mnangaku-tky@umin.ac.jp

Received 27 December 2010; revised 22 March 2011; accepted 16 April 2011
} 
renal replacement therapy. ${ }^{6}$ Indoxyl sulfate (IS) is a representative organic compound that has been proposed to contribute to the development of uremic symptoms. ${ }^{7}$ Our recent work demonstrated that IS induces endoplasmic reticulum stress and thereby contributes the progression of cellular damages in tubular epithelial cells. ${ }^{8}$ Clearly, IS appears to have an important role in the pathogenesis of renal diseases. ${ }^{9,10}$

Based on these previous reports, we decided to connect the possible links of uremic toxins to the desensitization of the oxygen-sensing mechanism in EPO-producing cells. Here we investigated the effect of IS on EPO expression in HepG2 cells in vitro, and of transient IS accumulation on renal EPO expression in rats in vivo.

\section{MATERIALS AND METHODS Cell Culture}

The human hepatoma cell line HepG2 was obtained from RIKEN BioResource Center (Tsukuba, Japan). Cells were maintained in Dulbecco's modified Eagle's medium (DMEM; Nissui Seiyaku, Tokyo, Japan) at pH 7.4, containing 10\% fetal calf serum (FCS; SAFH Biosciences, Lenexa, KS). Cells were cultured in humidified $95 \%$ air with $5 \%$ carbon dioxide at $37^{\circ} \mathrm{C}$. Cells received fresh medium $12 \mathrm{~h}$ before nuclear extract preparation and RNA isolation, and $16 \mathrm{~h}$ before transient transfection. Unless otherwise noted, IS (Sigma), indoleacetic acid (IAc; WAKO Pure Chemical Industries, Osaka, Japan) and the cobalt chloride $\left(\mathrm{CoCl}_{2}\right)$ (Sigma) were added to the media with serum at final concentrations of $1 \mathrm{mM}$ and $100 \mu \mathrm{M}$, respectively.

\section{Lactate Dehydrogenase (LDH) Assay}

After removal of the medium, treated cells in a 24-well plate were lysed with $0.5 \%$ Triton X-100 and incubated at room temperature for $30 \mathrm{~min}$. LDH concentration of medium and cell lysates was measured using LDH Kainos (Kainos Laboratories, Tokyo, Japan) according to the manufacturer's protocol. The ratio of medium LDH to the sum of medium LDH and cellular LDH was used as a marker of cellular injury. ${ }^{8}$

\section{MTS Assay}

To test the cell viability, we performed an MTS assay, using CellTiter 96 AQueous One Solution (Promega, Madison, WI) according to the manufacturer's protocol. Briefly, $5 \times 10^{3}$ HepG2 cells were grown in $100 \mu$ l of medium on 96-well plates for overnight. After they were treated as indicated for $24 \mathrm{~h}, 20 \mu \mathrm{l}$ of MTS reagent was added to each well of the 96well plate. After incubation at $37^{\circ} \mathrm{C}$ for $1 \mathrm{~h}$, the absorbance at $492 \mathrm{~nm}$ was measured using a plate reader (Asys Hitech, Eugendorf, Austria). ${ }^{8}$

\section{RNA Isolation and Quantitative Real-time PCR}

Total RNA was isolated by RNAiso plus (Takara, Tokyo, Japan) according to the manufacturer's protocol, reverse-
Table 1 Primers used for quantitative PCR

\begin{tabular}{lll}
\hline Primer & Forward: $5^{\prime}-3^{\prime}$ & Reverse: $5^{\prime}-3^{\prime}$ \\
\hline Rat EPO & TACGTAGCCTCACTTCACTGCTT & GCAGAAAGTATCCGCTGTGAGTGTTC \\
Rat actin & CTITCTACAATGAGCTGCGTG & TCATGAGGTAGTCTGTCAGG \\
Human EPO & AGGCCGAGAATATCACGACG & CCATCCTCTTCCAGGCATAGAAA \\
Human actin & TCCCCCAACTTGAGATGTATGAAG & AACTGGTCTCAAGTCAGTGTACAGG
\end{tabular}

Abbreviation: EPO, erythropoeitin.

transcribed and the amount of mRNAs was evaluated by quantitative real-time PCR. cDNA was synthesized using ImProm-II reverse transcription system (Promega), from $1 \mu \mathrm{g}$ template RNA in a $20 \mu \mathrm{l}$ reaction volume. One microliter of cDNA was added to Thunderbird qPCR Mix (Toyobo) and subjected to PCR amplification (cycled 40 times at $95^{\circ} \mathrm{C}$ for $30 \mathrm{~s}, 60^{\circ} \mathrm{C}$ for $30 \mathrm{~s}$ and $72^{\circ} \mathrm{C}$ for $30 \mathrm{~s}$ ) in the iCycler system (Bio-Rad). We employed $\beta$-actin as an internal control. PCR was conducted in triplicate for each sample. The sequences of primers used for human and rat EPO and $\beta$-actin mRNA are shown in Table $1 .^{11}$

\section{Nuclear Extract Preparation}

Nuclear proteins were extracted and isolated from HepG2 cells using the Thermo Scientific NE-PER Nuclear and Cytoplasmic Extraction Kit protocol, after adding protease inhibitor cocktail (Roche Applied Science, Indianapolis, IN). Briefly, cells were washed with ice-cold phosphate-buffered saline (PBS) and centrifuged at $500 \mathrm{~g}$ and $4{ }^{\circ} \mathrm{C}$ for $5 \mathrm{~min}$. The cells were lysed in cytoplasm extraction reagent I (CER I) for $10 \mathrm{~min}$, then followed by (CER II) for $1 \mathrm{~min}$, and spun at $16000 \mathrm{~g}$ to extract the cytoplasmic proteins. Proteins from the nuclear material were then extracted by adding nuclear extraction reagent to the nuclei with vigorous vortex per $10 \mathrm{~min}$ for four times, and spun at $16000 \mathrm{~g}$. Nuclear extracts were stored at $80^{\circ} \mathrm{C}$ until used. Protein concentrations were determined using the Bradford method with bovine serum albumin as standard.

\section{Western Blot Analyses}

For determination of HIF- $1 \alpha$ and HIF- $2 \alpha$ protein, $30 \mu \mathrm{g}$ nuclear extract protein was loaded in each lane. These protein samples were separated by electrophoresis on an $8 \%$ SDS-polyacrylamide gel, followed by electrotransfer to polyvinylidene difluoride membranes (GE Healthcare BioSciences, Little Chalfont, UK). The transferred membranes were blocked with $5 \%$ fat-free skim milk in tris-buffered saline with $0.01 \%$ Tween 20 for $60 \mathrm{~min}$ at room temperature. The membrane was incubated with rabbit polyclonal anti-HIF- $1 \alpha$ or HIF- $2 \alpha$ (diluted 1:1000 in PBS/5\% skim milk; Novus. Biologicals) antibody overnight at $4{ }^{\circ} \mathrm{C}$. HRPconjugated anti-rabbit IgG (Promega) was then used as the 
secondary antibody. Immunoreactive protein was visualized by the chemiluminescence protocol (ECL, GE Healthcare Bio-Sciences). Anti-actin rabbit polyclonal antibody (1:1000; Sigma-Aldrich) was used for calibration. ${ }^{12}$

\section{Luciferase Assays}

The hypoxia-responsive reporter vector with tandem copies of the hypoxia-responsive element (HRE) from the rat vascular endothelial growth factor gene subcloned into $5^{\prime}$ region of hmCMV-promoter-luciferase transcription unit (pHREluc) was constructed in our previous work. ${ }^{13}$ Five-hundred nanogram of pHRE-luc and $25 \mathrm{ng}$ of pTK-renilla luciferase (Promega) per $1 \times 10^{5}$ HepG2 cells were co-transfected using Lipofectamine LTX with Plus reagent (Invitrogen) in 24-well culture dishes. The transfected cells were exposed to $\mathrm{CoCl}_{2}(100 \mu \mathrm{M})$ for the indicated time and harvested by $150 \mu$ l of passive protein lysis buffer for dual luciferase assay.
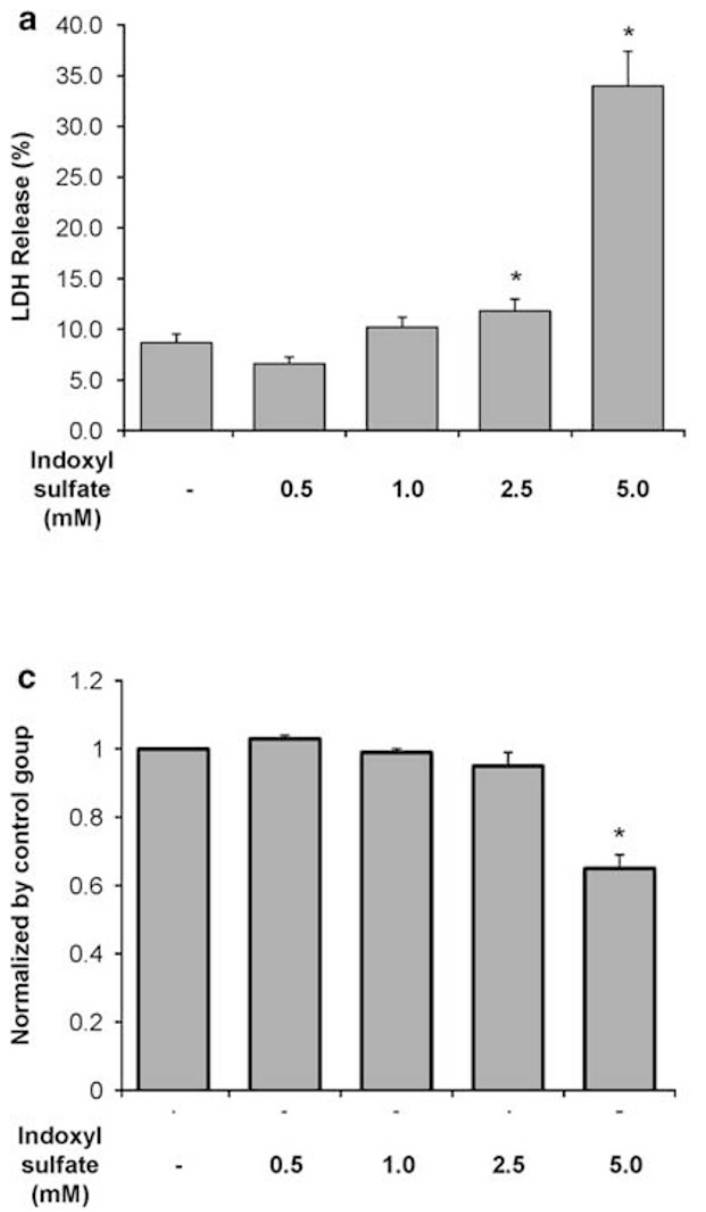

A Lumat 9507 luminometer (EG and Berthold, Bad Wildbad, Germany) was used for measurement. In order to correct for transfection efficiency, the relative value of the firefly luciferase light unit was divided by that of the renilla luciferase.

\section{Animal Experiments}

We obtained male Sprague-Dawley rats at the age of 8 weeks from Japan Charles River (Yokohama, Japan). After $20 \mathrm{~h}$ of starvation, $60 \mathrm{mg} / \mathrm{kg}$ of $\mathrm{CoCl}_{2}$ (Sigma), or vehicle (distilled water), was given via subcutaneous injection, with or without administration of indole $(10 \mathrm{mg} / \mathrm{kg}$; Merck, Whitehouse Station, NJ) in $0.5 \%$ methyl cellulose (Wako Pure Chemical Industries) by gavage. ${ }^{14}$ Ten hours after indicated treatment, rats were killed. The renal cortex was harvested, and fixed in formalin or stocked at $-80^{\circ} \mathrm{C}$ until analyzed. Histological analysis was performed by hematoxylin and eosin staining.
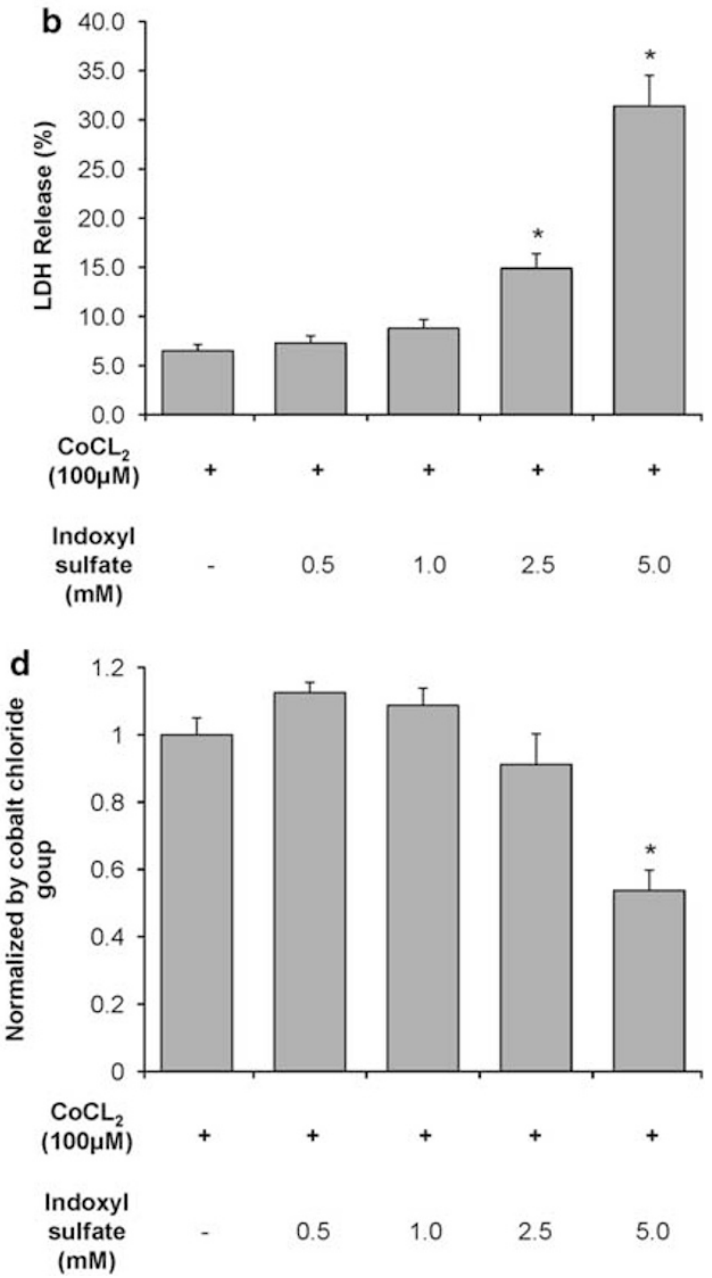

Figure 1 Effects of indoxyl sulfate (IS) treatment on cell viability in HepG2 cells. The cell viability of HepG2 treated with IS with and without $100 \mu$ M cobalt chloride $\left(\mathrm{CoCl}_{2}\right)$ was analyzed by lactate dehydrogenase (LDH) release assay (a and b) and MTS assay (c and d). (a) Five mM and $2.5 \mathrm{mM} \mathrm{IS-induced}$ significant LDH release as compared with the untreated group. (b) As compared with $\mathrm{CoCl}_{2}$-treated group, co-administration with 2.5 and $5.0 \mathrm{mM}$ IS significantly enhanced LDH release in HepG2 cells. (c) HepG2 cell viability was significantly decreased by $5.0 \mathrm{mM}$ IS. (d) In combination with CoCl 2 treatment, $5.0 \mathrm{mM}$ IS further reduces cell viability. $n=3$ for each in three to four dependent experiments. ${ }^{*} P<0.05$ vs control group. 
All animal experiments were performed in accordance with the National Institutes of Health guidelines for use and care of laboratory animals, and approved by the local ethical committees.

\section{Measurement of Plasma EPO Protein}

We used cardiac puncture blood sampling after rats were killed. Blood samples were immediately mixed within the EDTA tube. Blood plasma was collected and immediately centrifuged at $3000 \mathrm{rpm}$ for $10 \mathrm{~min}$, and plasma was stored at $-80^{\circ} \mathrm{C}$ until analyzed. Plasma EPO levels were determined by enzyme-linked immunosorbent assays using Quantikine Mouse/Rat Epo Immunoassay kit (MEP00; R\&D Systems, Minneapolis, MN).

\section{Statistical Analysis}

The values given in this article are presented as mean \pm s.e.m. All analyses were performed by analysis of variance, followed by a Fisher's least significant difference. All statistics calculations were done with SPSS 16.0 for Windows.

a

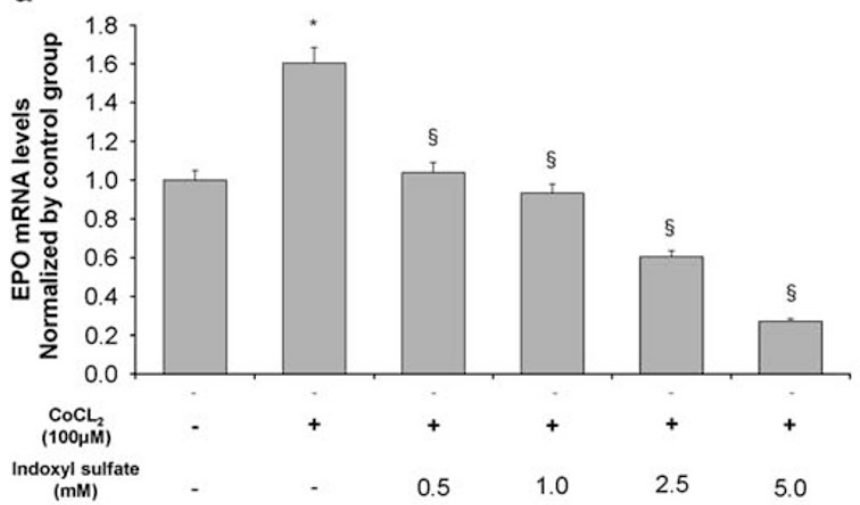

\section{RESULTS}

\section{IS Induces HepG2 Cellular Injury}

To assess the effects of IS on viability of HepG2 cells, we performed $\mathrm{LDH}$ assay in which $\mathrm{LDH}$ leakage from cells into medium is used as a marker of cellular damage. After $24 \mathrm{~h}$ treatment of IS, we observed significant LDH leakage at 2.5 and $5.0 \mathrm{mM}$ of IS, as compared with untreated cells (Figure 1a). Furthermore, treatment of $\mathrm{CoCl}_{2}$, as a hypoxia mimetic, did not aggravate cellular damage induced by IS (Figure 1b). We further investigated the non-lethal concentration of IS by MTS assay, to exclude the possibility that the findings in the following experiments reflect cellular toxicity rather than a specific effect, thus allowing to explore the possible impact of IS on EPO regulation. Twenty-four hour treatment with IS resulted in significant viability suppression at $5.0 \mathrm{mM}$ (Figure $1 \mathrm{~b}, 65 \pm 4 \%$ as compared with control group, $P<0.05$; Figure 1c). As compared with $\mathrm{CoCl}_{2}$ treatment alone, combination of $\mathrm{CoCl}_{2}$ and IS treatment revealed further suppression of viability at $5.0 \mathrm{mM}$ of IS (Figure 2b, $54 \pm 6 \%, P<0.05$; Figure 1d). Taken together, this

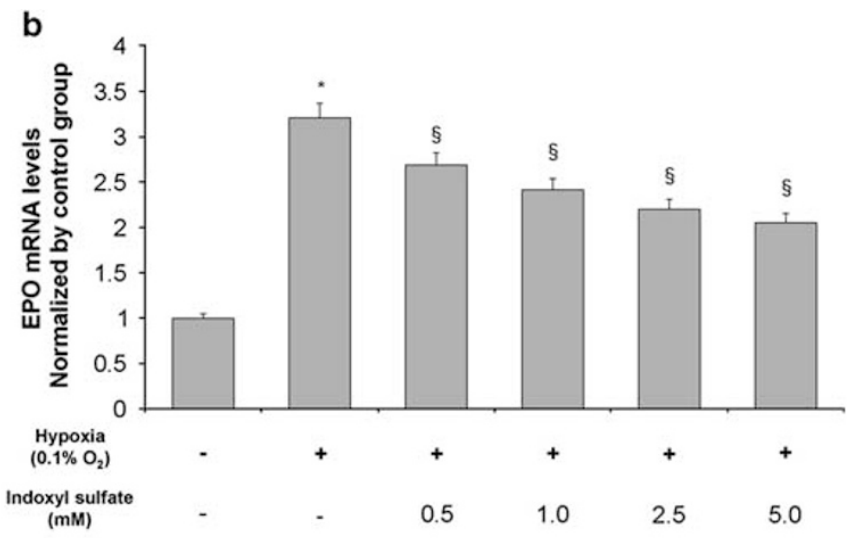

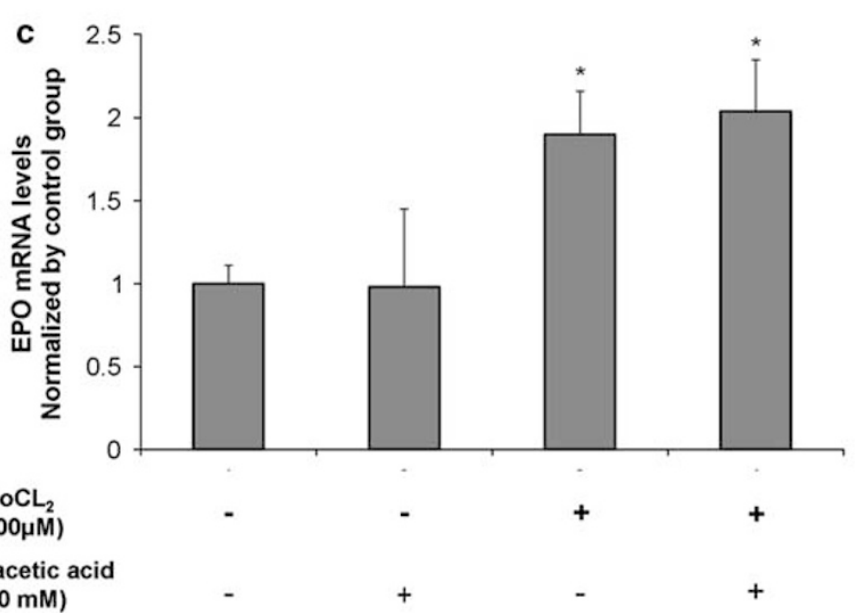

Figure 2 Indoxyl sulfate (IS) suppressed cobalt chloride $\left(\mathrm{CoCl}_{2}\right)$ - and hypoxia-induced erythropoietin (EPO) mRNA expression in a dose-dependent manner in HepG2 cells. Real-time quantitave PCR was performed to assess the change in EPO mRNA expression in HepG2 cells treated with IS under hypoxic condition. (a) $\mathrm{CoCl}_{2}$-treated HepG2 cells enhanced EPO mRNA expression by 1.60 fold. Three hours of preincubation with IS significantly suppressed $\mathrm{CoCl}_{2}$-induced EPO mRNA expression. (b) Hypoxia induced 3.21-fold increase in EPO mRNA expression. (c) Incubation with indoleacetic acid (IAc) suppressed $\mathrm{CoCl}_{2}$-induced EPO mRNA expression. IS significantly suppressed hypoxia-induced EPO mRNA expression. $n=6$ for each, ${ }^{\star} P<0.05$, as compared with untreated group; ${ }^{\S} P<0.05$, as compared with the cells treated with $\mathrm{CoCl}_{2}$ or hypoxia alone. 
illustrates that high concentration of IS has toxicity in EPO producing HepG2 cells. In further studies, we focused on the effects of IS at the concentrations of $1.0 \mathrm{mM}$ and below.

\section{IS Suppresses EPO mRNA Expression in HepG2 Cells}

To further explore the effects of IS on EPO mRNA expression, we exposed HepG2 cells to $\mathrm{CoCl}_{2}$ with various concentrations of IS, and examined EPO mRNA expression by real-time quantitative PCR analysis. As shown in Figure 2a, IS dose-dependently suppressed $\mathrm{CoCl}_{2}$-induced EPO mRNA expression. IS also demonstrated the similar suppressive effects on EPO mRNA in hypoxic $\left(0.1 \% \mathrm{O}_{2}\right)$ conditions (Figure 2b). In contrast, IAc failed to suppress EPO expression (Figure 2c). These results clearly show that IS suppresses EPO mRNA expression induced by hypoxia or hypoxia mimetics.

\section{IS Decreases Nuclear Accumulation of Hypoxia-Induced Factors in HepG2 Cells}

It is well known that HIF has the major role in hypoxic induction of EPO. Therefore, we measured the amounts of the nuclear fraction of HIF- $1 \alpha$ and HIF- $2 \alpha$ in $\mathrm{CoCl}_{2}$-stimulated HepG2 cells. As shown in Figure 3, $\mathrm{CoCl}_{2}$ significantly induced the accumulation of HIF- $1 \alpha$ and HIF- $2 \alpha$ proteins in the nuclear fraction by 8.4 and 2.8 folds, as compared with the untreated cells under normoxia, whereas they were decreased in the presence of IS $(1.0 \mathrm{mM})$ in HIF1- $\alpha$ and HIF2- $\alpha$ proteins by 3.3 and 2.1 folds, respectively (Figure 3 ). To test whether a decrease in HIF- $\alpha$ accumulation was functional, we arranged HRE-luciferase assay (Figure 4). Although $\mathrm{CoCl}_{2}$ induced HRE-driven luciferase activity by 2.40 fold, IS $(1.0 \mathrm{mM})$ significantly dampened induction to 1.25 fold. These results raise the possibility that the impaired production of EPO in hypoxia may have been mediated by dysregulated nuclear accumulation of HIFs- $\alpha$ and impaired transcriptional activity of HIFs.

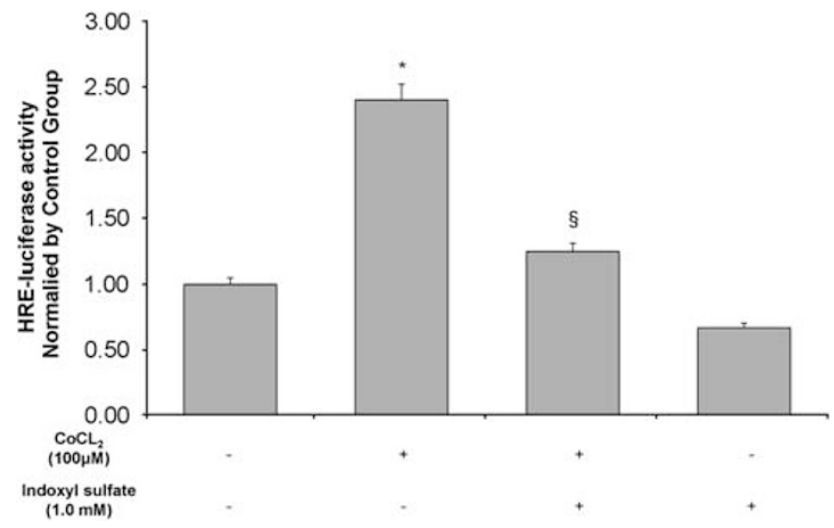

Figure 4 Indoxyl sulfate (IS) suppressed cobalt chloride $\left(\mathrm{CoCl}_{2}\right)$-induced hypoxia-responsive element (HRE)-luciferase activity in HepG2 cells. HepG2 cells were treated with $\mathrm{CoCl}_{2}$ in the presence or absence of IS and then HRE-luciferase assay was performed. $\mathrm{CoCl}_{2}$-treated HepG2 cells enhanced HRE-luciferase activity by 2.4-fold as compared with control group. Three hours of preincubation with IS significantly suppressed $\mathrm{CoCl}_{2}$-induced HRE-luciferase activity to 1.25 -fold of the basal level. $n=3$ for each, ${ }^{\star} P<0.05$, as compared with control group; ${ }^{\S} P<0.05$, as compared with the cells treated with $\mathrm{CoCl}_{2}$ alone. a
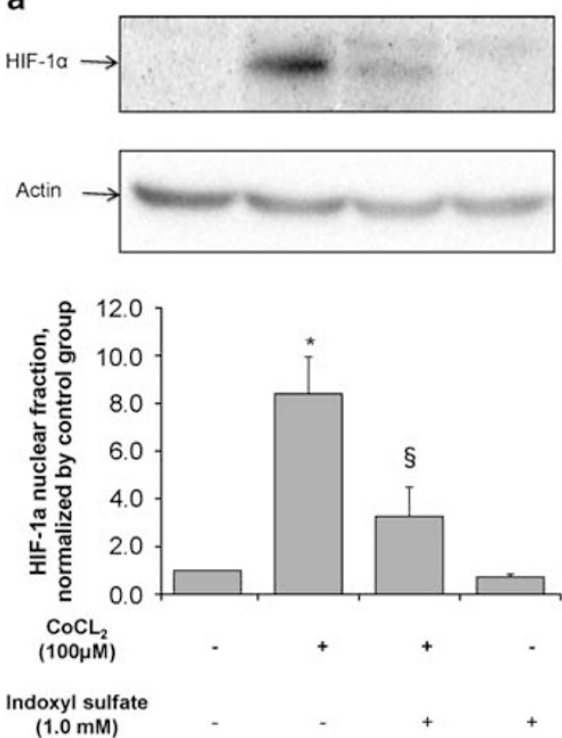

b
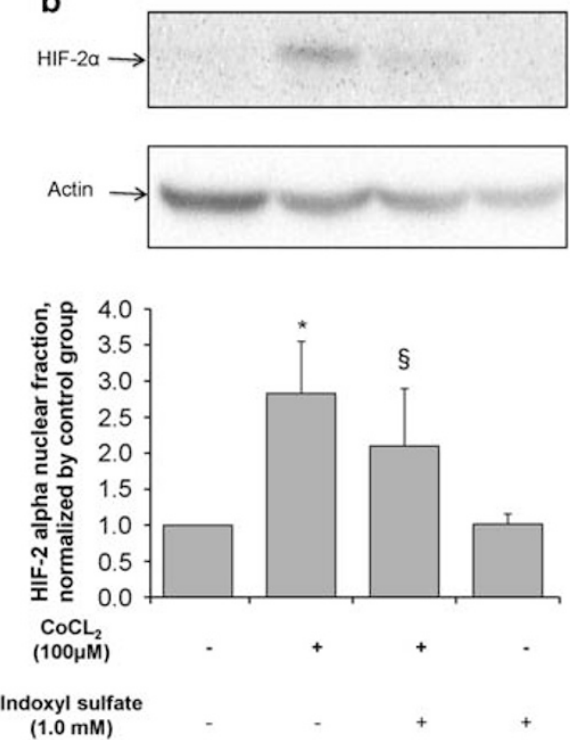

Figure 3 Indoxyl sulfate (IS) decreased nuclear fraction of both hypoxia-inducible transcription factor (HIF)- $1 \alpha$ and HIF- $2 \alpha$ in HepG2 cells. HepG2 cells were treated with cobalt chloride $\left(\mathrm{CoCl}_{2}\right)$ in the presence or absence of IS and the amount of HIF-1 $\alpha(\mathbf{a})$ and HIF- $2 \alpha(\mathbf{b})$ in the nuclear fraction were analyzed by western blot analysis, followed by densitometry. $\mathrm{CoCl}_{2}$-treated $\mathrm{HepG} 2$ cells increased both the nuclear fraction of $\mathrm{HIF}-1 \alpha$ by $8.4-$ fold, and HIF- $2 \alpha$ by 2.8 -fold as compared with control group. Three hours of preincubation with IS significantly suppressed the $\mathrm{CoCl}_{2}$-induced nuclear fraction of both HIF- $1 \alpha$ and HIF- $2 \alpha$ to 3.3- and 2.1-fold, respectively. $n=6$ for each, ${ }^{\star} P<0.05$, as compared with control group; ${ }^{\S} P<0.05$, as compared with the group treated with $\mathrm{CoCl}_{2}$ alone. 


\section{Decreased EPO mRNA of the Kidney and EPO Plasma Levels in Animals Treated with Indole}

Next, we examined whether an increase in serum IS concentrations suppresses renal EPO mRNA and plasma EPO levels in vivo. As shown in Figure 5a, EPO mRNA expression was robustly induced by subcutaneous injection of $\mathrm{CoCl}_{2}(60 \mathrm{mg} / \mathrm{kg})$ by 420 times, and oral gavage of indole $(10 \mathrm{mg} / \mathrm{kg})$ significantly suppressed the induction to 124 times as compared with control rats $(P<0.05)$. We further evaluated the plasma EPO levels of the experimental animals (Figure 5b). In the normal control rats, the EPO plasma level was approximately $10 \mathrm{pg} / \mathrm{ml}$. Subcutaneous $\mathrm{CoCl}_{2}$ injection induced a significant increase in circulating EPO to $5486 \pm 1097 \mathrm{pg} / \mathrm{ml}$, and indole treatment suppressed $\mathrm{CoCl}_{2}$-induced plasma EPO levels to $2039 \pm 449 \mathrm{pg} / \mathrm{ml}$. These data fully support that IS suppresses the EPO production in vivo. Administration of indole at this dose did not induce any parenchymal damage of the kidney (Figure 6).

\section{DISCUSSION}

Results of the present study clearly indicated that IS induced HepG2 cellular injury at relatively high concentrations (more than $2.5 \mathrm{mM})$. On the other hand, IS-treated HepG2 cells impaired $\mathrm{CoCl}_{2}$ - and hypoxia-induced EPO mRNA expression in a dose-dependent manner, at concentrations below toxic ranges (Figure 2). IAc at the equivalent concentration failed to suppress EPO production, suggesting a specific effect of IS. The suppression of EPO gene expression by IS was associated with a decrease in the nuclear fraction of HIFs- $\alpha$, which was associated with reduction of HRE-driven luciferase activity in HepG2 cells. These in vitro findings were confirmed by in vivo observations that administration of indole led to suppression of renal EPO mRNA expression and plasma EPO levels in $\mathrm{CoCl}_{2}$-treated rats. Serum levels of IS in non-dialysis patients with CKD were reported to be $0.08 \mathrm{mM} .{ }^{15}$ In our previous study, we observed an increase in oxygen consumption with IS at the concentrations of $0.1 \mathrm{mM}$ in rat tubular cells and $0.15 \mathrm{mM}$ in rat kidneys. In the present study, we used $40 \%$ of the dosage of indole as compared with our previous works, ${ }^{14}$ to mimic the mild elevation of serum IS in the CKD patients. Thus, the effects of IS on renal EPO production in vivo were observed within a pathophysiological range of uremic states. The results of histological analysis showed no parenchymal injury of the kidney, emphasizing a
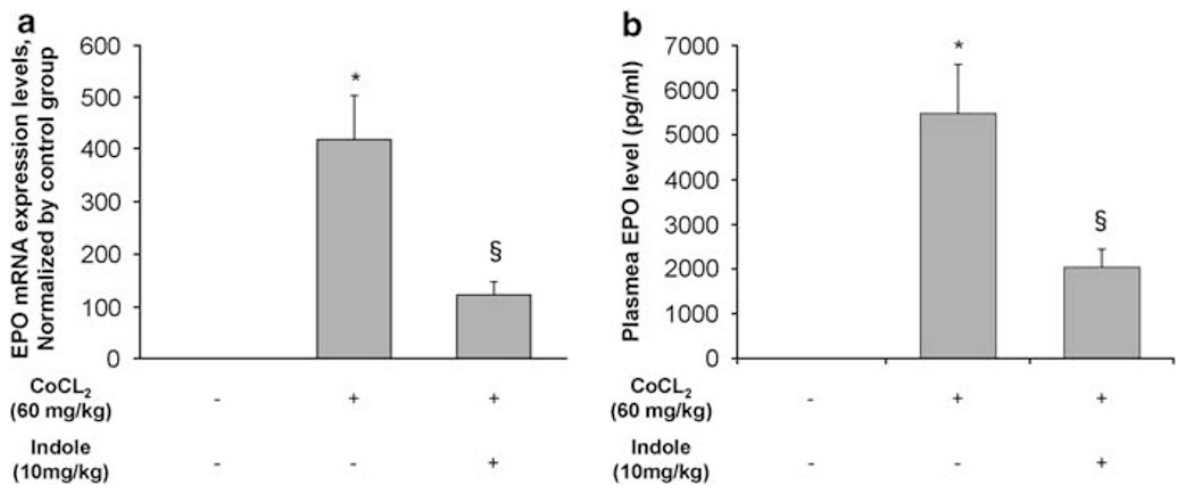

Figure 5 Indoxyl sulfate (IS) suppressed cobalt chloride $\left(\mathrm{CoCl}_{2}\right)$-induced erythropoietin (EPO) mRNA expression in the kidney and plasma EPO levels in vivo. (a) Subcutaneous injection of $\mathrm{CoCl}_{2}(60 \mathrm{mg} / \mathrm{kg})$ induced elevations of renal EPO mRNA levels, estimated by real-time quantitative PCR, by 420 -fold as compared with untreated control rats. Gavage administration of indole $(10 \mathrm{mg} / \mathrm{kg})$ significantly suppressed $\mathrm{CoCl}_{2}$-induced renal EPO mRNA expression to 124 -fold of the basal level. (b) Indole $\left(10 \mathrm{mg} / \mathrm{kg}\right.$ ) treatment suppressed plasma EPO levels of $\mathrm{CoCl}_{2}$-treated rats from 5486 to $2039 \mathrm{pg} / \mathrm{ml}$. $n=3$ for each, ${ }^{\star} P<0.05$, as compared with control group; ${ }^{\circledR} P<0.05$, as compared with the group treated with $\mathrm{CoCl}_{2}$ alone.
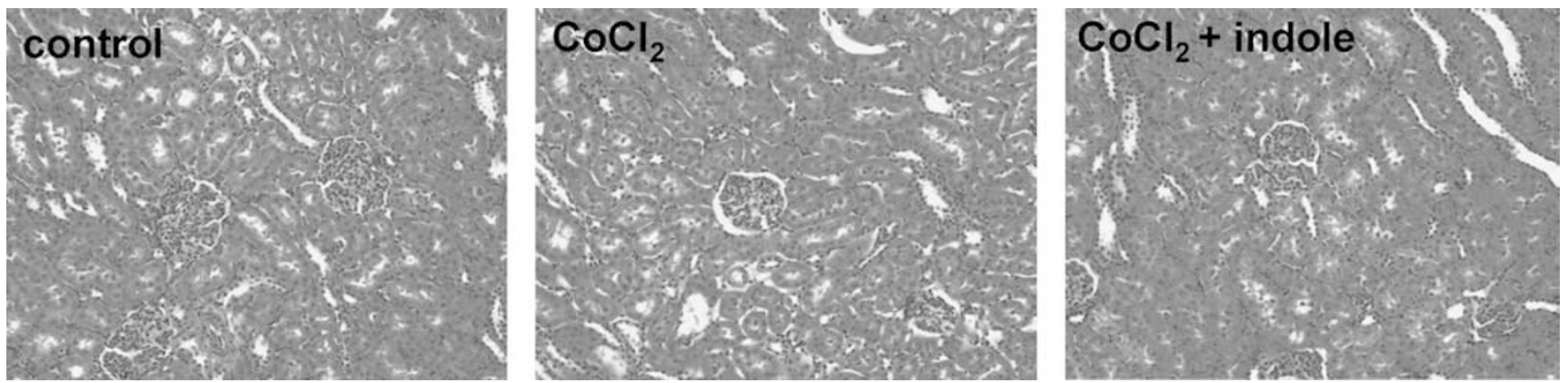

Figure 6 Hematoxylin and eosin staining of the experimental animals. No parenchymal damage was observed in cobalt chloride $\left(\mathrm{CoCl}_{2}\right)$-treated animals with and without indole. $\times 200$. 
functional effect of uremia on renal EPO expression. Our study, for the first time, provided evidence that links IS, a representative uremic toxin, to impairment of EPO production through HIF-dependent oxygen-sensing mechanism both in vitro and in vivo.

According to the works by Bernhardt et al, ${ }^{5}$ pharmacological activation of HIFs in dialysis patients significantly induced renal EPO production, which was equivalent to the doses of EPO used to maintain target hemoglobin levels recommended by the guideline. ${ }^{16}$ The inability to produce EPO by renal interstitial EPO-producing cells, presumably as a result of desensitization of the oxygen-sensing mechanism rather than destruction of EPO-producing cells, seems to cause the inappropriately low production of the hormone in patients with CKD. Several observational studies showed that plasma EPO concentrations and erythropoiesis index in dialysis patients can be enhanced in response to acute episodes of hypoxia and hemorrhage, despite not responding adequately to the chronic reduction in hemoglobin levels. ${ }^{17-19}$ In this work, we clearly demonstrate the possible connection between uremic status and inadequate synthesis of EPO.

To date, more than 90 molecules have been identified as uremic toxins, ${ }^{20}$ and IS is one of the most extensively studied. After protein-derived tryptophan is metabolized into indole by tryptophanase in intestinal bacteria, indole is delivered to the liver via portal circulation, and then further metabolized to IS in the liver. Our recent works demonstrated that IS dysregulates oxygen metabolism and enhances oxygen consumption in tubular cells, thus aggravating tubulointerstitial hypoxia, which could be reversed by an oral sorbent of uremic toxin (AST-120) in the remnant kidney. ${ }^{14}$ Theoretically, the interstitial hypoxia should facilitate recruitment of HIF to the HRE in the $3^{\prime}$-enhancer region of EPO genes. However, EPO synthesis in the uremic kidney is inadequate. Here, we hypothesized that accumulation of uremic toxins in EPO-producing cells might dampen hypoxia response, which might explain an inadequate EPO production in relatively severe hypoxic microenvironment. In this work, the nuclear fraction of HIFs- $\alpha$ and HRE luciferase activity were significantly reduced by IS in $\mathrm{CoCl}_{2}$-treated $\mathrm{HepG}_{2}$ cells (Figure 3). The detailed mechanism of this phenomenon is still unclear. Nevertheless, results of the present study can partly explain that why the EPO-producing cells respond inappropriately to severe hypoxia in the uremic status.

One limitation of our study is that we used an acute animal model of high IS levels to study the effects of CKD on EPO production. Because our main purpose was to investigate a functional consequence of uremia, we used an acute model in order to exclude parenchymal kidney damage as a confounding factor observed in animal models of CKD such as the remnant kidney. Another limitation is that we used only non-renal EPO producing cells, namely the HepG2 cells. However, there are no renal EPO-producing cultured cells at this moment, although recent establishment of transgenic mouse lines expressing green fluorescent protein under the control of a $180-\mathrm{kb}$ mouse Epo gene locus will enable us to isolate renal EPO-producing cells in the future. $^{21}$

In conclusion, we demonstrated for the first time that IS, a representative uremic toxin, has a pathologic role in inappropriate EPO production by disturbing the oxygensensing system both in vitro and in vivo, by suppressing nuclear translocation of HIF- $\alpha$ protein and transcriptional activity of HIF thereof. Antagonism of the suppressing effects of IS may facilitate HIFs availability and represent a novel therapeutic approach in renal anemia.

\section{ACKNOWLEDGEMENT}

This work was supported by Grant-in-Aids for Scientific Research 19390228 and 2139036 (to MN) from the Japan Society for the Promotion of Science and research grant from The Kidney Foundation, Japan (JKFB11-2).

\section{DISCLOSURE/CONFLICT OF INTEREST}

The authors declare no conflicts of interest.

1. Hsu CY, McCulloch CE, Curhan GC. Epidemiology of anemia associated with chronic renal insufficiency among adults in the United States: Results from the Third National Health and Nutrition Examination Survey. J Am Soc Nephrol 2002;13:504-510.

2. Kohagura K, Tomiyama N, Kinjo $\mathrm{K}$, et al. Prevalence of anemia according to stage of chronic kidney disease in a large screening cohort of Japanese. Clin Exp Nephrol 2009;13:614-620.

3. Mimura I, Nangaku M. The suffocating kidney: tubulointerstitial hypoxia in end-stage renal disease. Nat Rev Nephrol 2010;6:667-678.

4. Tanaka T, Nangaku M. The role of hypoxia, increased oxygen consumption, and hypoxia-inducible factor- 1 alpha in progression of chronic kidney disease. Curr Opin Nephrol Hypertens 2010; 19:43-50.

5. Bernhardt WM, Wiesener MS, Scigalla $P$, et al. Inhibition of prolyl hydroxylases increases erythropoietin production in ESRD. J Am Soc Nephrol 2010;21:2151-2156.

6. de Groot K, Bahlmann FH, Sowa J, et al. Uremia causes endothelial progenitor cell deficiency. Kidney Int 2004;66:641-646.

7. Niwa T. Uremic toxicity of indoxyl sulfate. Nagoya J Med Sci 2010; 72:1-11.

8. Kawakami T, Inagi R, Wada T, et al. Indoxyl sulfate inhibits proliferation of human proximal tubular cells via endoplasmic reticulum stress. Am J Physiol Renal Physiol 2010;299:F568-F576.

9. Wu IW, Hsu KH, Lee CC, et al. p-Cresyl sulphate and indoxyl sulphate predict progression of chronic kidney disease. Nephrol Dial Transplant 2011;26:938-947.

10. $\mathrm{Yu} M, \mathrm{Kim} \mathrm{YJ}$, Kang DH. Indoxyl sulfate-induced endothelial dysfunction in patients with chronic kidney disease via an induction of oxidative stress. Clin J Am Soc Nephrol 2011;6:30-39.

11. Mimura I, Nangaku $M$, Nishi $H$, et al. Cytoglobin, a novel globin, plays an antifibrotic role in the kidney. Am J Physiol Renal Physiol 2010;299:F1120-F1133.

12. Kojima I, Tanaka T, Inagi R, et al. Metallothionein is upregulated by hypoxia and stabilizes hypoxia-inducible factor in the kidney. Kidney Int 2009;75:268-277.

13. Tanaka $T$, Miyata $T$, Inagi $R$, et al. Hypoxia in renal disease with proteinuria and/or glomerular hypertension. Am J Pathol 2004;165:1979-1992.

14. Palm F, Nangaku M, Fasching A, et al. Uremia induces abnormal oxygen consumption in tubules and aggravates chronic hypoxia of the kidney via oxidative stress. Am J Physiol Renal Physiol 2010;299: F380-F386.

15. Niwa T, Miyazaki T, Tsukushi $S$, et al. Accumulation of indoxyl-betaD-glucuronide in uremic serum: suppression of its production by oral sorbent and efficient removal by hemodialysis. Nephron 1996; 74:72-78. 
16. $\mathrm{K} / \mathrm{DOQ}$ I clinical practice guidelines for cardiovascular disease in dialysis patients. Am J Kidney Dis 2005;45(4 Suppl 3):S1-S153.

17. Walle AJ, Wong GY, Clemons GK, et al. Erythropoietin-hematocrit feedback circuit in the anemia of end-stage renal disease. Kidney Int 1987;31:1205-1209.

18. Chandra M, Clemons GK, McVicar MI. Relation of serum erythropoietin levels to renal excretory function: evidence for lowered set point for erythropoietin production in chronic renal failure. J Pediatr 1988;113: 1015-1021.
19. Blumberg A, Keller H, Marti HR. Effect of altitude on erythropoiesis and oxygen affinity in anaemic patients on maintenance dialysis. Eur J Clin Invest 1973;3:93-97.

20. Vanholder R, De Smet R, Glorieux $G$, et al. Review on uremic toxins: classification, concentration, and interindividual variability. Kidney Int 2003;63:1934-1943.

21. Obara N, Suzuki N, Kim K, et al. Repression via the GATA box is essential for tissue-specific erythropoietin gene expression. Blood 2008;111:5223-5232. 